\title{
Labyrinthe
}

36 | 2011 (1)

«Par les Grecs »

\section{Énième hellénique}

\section{Laurent Dubreuil}

\section{OpenEdition}

\section{Journals}

\section{Édition électronique}

URL : http://journals.openedition.org/labyrinthe/4113

DOI : 10.4000/labyrinthe.4113

ISSN : 1950-6031

\section{Éditeur}

Hermann

\section{Édition imprimée}

Date de publication : 15 février 2011

Pagination : 13-30

ISBN : 9782705680541

\section{Référence électronique}

Laurent Dubreuil, «Énième hellénique », Labyrinthe [En ligne], 36 | 2011 (1), mis en ligne le 01 février 2013, consulté le 21 avril 2019. URL : http://journals.openedition.org/labyrinthe/4113; DOI : 10.4000/ labyrinthe. 4113

Propriété intellectuelle 


\section{Énième hellénique}

Laurent DUBREUIL

Contact:1d79@cornell.edu

[1] Les Grecs, vraiment? En êtes-vous sûr? S'agit-il d'un retour salutaire? à éviter? Et qui est-ce d'abord, et de qui et de quoi parlezvous? Même, le parlez-vous? je veux dire parlez-vous grec quand du moins vous parlez d'eux? Qu'avez-vous d'ailleurs à défendre qu'eux à travers eux, et d'autres (et elles?); ou vous mettez-vous en quête de notre identité non-nationale; ou bien cherchez-vous plus simplement à rétablir quelque chose, comme par hasard, par chance, par fatalité? Toutes ces questions m'assaillent, qui contiennent ou provoquent nombre d'interrogations supplémentaires, car s'il faut encore penser aux vieux Grecs aujourd'hui - or il le faut, semble-t-il, dans un temps où la philosophie contemporaine bruit toujours de mots helléniques talismaniques, où la scène politique continue de se saturer en certains de ses bords d'une défense quasi spartiate de l'Occident, où la nouvelle troisième dimension du cinéma s'ouvre illico à l'antique mythologie, où surabondent les traductions des plus obscurs ouvrages rédigés en grec ancien -, alors moins que jamais nous ne pouvons ignorer l'ampleur des revendications, des domestications, des oukases, qui firent et continuent de faire des " Grecs » un sujet impossible et nécessaire à notre penser. Il serait beau cependant de songer un tout autre rapport, précisément délié, suspendant la quête de l'ultime vérité comme l'encombrement de la tradition. Un peu naïf aussi puisque le philhellénisme n'est aucun sentiment spontané, que, donc, l'admiration ou l'intérêt ne jaillissent pas de rien, qu'ainsi les œuvres les suscitant furent bien conservées, et offertes. Oui, voilà ce sur quoi nous allons échanger. Ce texte voudrait se situer dans le champ de forces que véhiculent ces doutes et questions, réponses ou évidences, et que traverse innocent le rêve d'une mise en suspens, d'une hellénisation discursive qui ne serait pas pro-grecque.

[2] Que ces choses grecques que nous commençons d'évoquer soient aussi révolues, assurément. Qu'en ce sens elles apparaissent sur fond d'une, et surtout de plusieurs, histoire(s), certes. La première nécessité consiste toutefois à relever (puis contester) que ce qui nous reste de la 
Grèce ancienne, quand il est agi ou saisi au présent, est très largement donné comme déterminé par l'histoire. C'est-à-dire: oui, je reconnais un hier, et plus que cela, mais dois-je aussitôt estimer que cette position dans le temps reculé conditionne notre réflexion? Pour faire simple, se rallient au déterminisme la plupart de ceux qui, du vingt au vingt-etunième siècles, se mirent à causer Grecs. Ce large accord m'étonne, il m'arrête, et j'en vois soudain trois côtés : le goût de l'originaire, le souci du contexte, la passion du passéisme. Si je continue la métaphore topologique, ce sont potentiellement des dimensions, et les coordonnées de chaque livre, de chaque article déterminant « les Grecs » permettent plus d'une combinaison, avec tel étant fort haut dans l'origine et le passéisme mais au ras du zéro contexte, etc. Dans la simplification recherchée que je vais développer, et sans trop user si possible de rafales onomastiques, il s'ensuit qu'un côté n'empêche pas l'autre, quoique des incompatibilités existent. Avant de qualifier davantage, une remarque me brûle les lèvres, et je cède. Les Grecs, si nous désignons alors un ensemble unifié, n'existent pas, n'ont jamais existé. Répétons ce truisme, que les Grecs d'Homère ne sont pas ceux d'Alexandrie, ni ceux de l'Athènes classique (soit le panthéon minimal) ceux de Sparte au même moment, quant à l'écart entre hommes libres et autres habitants de la glorieuse Cité... Seulement, continuons d'avancer sur cette voie, et ajoutons: quand bien même des tendances sociales, des dispositions de civilisation, des manières, des paroles se retrouvent majoritairement sur telle durée dans ce lieu nommé Hellas, nous ne traitons ni avec les-Grecs, ni non plus avec leurs diverses manifestations empiriques et circonscrites (du style des Athéniens-sous-Pisistrate). Ce malgré les efforts antiques du panhellénisme, ce malgré les généralisations contemporaines de l'anthropologie historique. Pour prétendre, avec Michel Foucault et tant d'autres, que « les Grecs » ne pensaient pas avec « une pensée comme la nôtre $\rangle^{1}$, il faut admettre que nous savons qui nous sommes, et ce que nous pensons, et que les Grecs, fût-ce en lieu et milieu précis, s'accordaient entre eux. Toutes assertions insensées, invérifiables, invérifiées ${ }^{2}$.

1. Michel Foucault, Histoire de la sexualité, vol. II, Paris, Gallimard, 1984, p. 247.

2. Dans une perspective différente mais évidemment proche, lire de Jacques Derrida « Nous autres Grecs », in Barbara Cassin dir., Nos Grecs et leurs modernes, Paris, Seuil, 1992. Derrida y exprime une « inquiétude [qui] ne concerne rien de moins que l'identité d'un référent proprement nommé "le Grec", "les Grecs" ou "la Grèce" » (p. 251). Pour faire vite, je ne suis toutefois pas sûr que le rapport critique de Derrida à Heidegger évite assez les chausse-trappes de l'origine. 


\section{Énième hellénique}

Au nom de l'entité Les-Grecs, il devient alors facile de parler de deux poteries, de lire un texte de Platon comme s'il émanait de la position dominante (qui est réelle, mais justement, qu'arrive-t-il aux devenirs minoritaires?), d'ajouter deux ou trois considérations approximatives, et l'on écrit un volume sur l'histoire de la sexualité. Sauf que le travail n'a pas débuté. Il se trouve que le succès de l'école historique autour de Jean-Pierre Vernant a fixé cette légende unificatrice des Grecs pour plusieurs générations de savants fascinés par une anthropologie de type conceptuel, qui juxtaposait l'artefact social à l'expression langagière et désenclavait du coup l'archéologie et les lettres classiques. Il s'en faut néanmoins que le bloc les-Grecs date des années dix-neuf cent soixante, et plus d'un Athénien de l'Antiquité s'employait déjà à y faire croire. D'où la tentation si répandue, dans les directions de la détermination historique, à postuler plus ou moins fermement les-Grecs. Autant la collusion n'est rien de sérieux, autant son évitement absolu n'est pas souhaitable, car le mécanisme d'une assimilation est souvent recelé par cette institution (comme la tragédie qui fait entendre sur scène des dialectes différents), ou secrété par ces échos textuels (d'Homère à Sophocle à Platon à Plutarque, par exemple). Au mieux, je n'ai donc affaire qu'à des Grecs, soit un ensemble demeurant hétérogène, voire contradictoire, un ensemble malgré tout, que je ne contribue pas moins à circonscrire que la supposée unité de civilisation. Poser ce point sans plus attendre pourrait revenir à relativiser par avance les faces de la détermination historique. Ce ne serait pas une juste exposition, mais tant pis; il reste dans tous les cas beaucoup à dire.

[3] Ce que je fais ici, à bride abattue. Et d'une appropriation supplémentaire: l'origine est grecque, la Grèce est l'origine; pardi. Vous n'ignorez pas ces mots, d'étymologie grecque, vrai, et qui servent de repère dans notre vie: poésie, philosophie, théâtre, mathématique, politique, logique, démocratie, physique. Ces mots parlent grec en vous et confient qu'alors tout commença ${ }^{3}$. La preuve par le nom vaut ce qu'elle vaut, on s'en aperçoit parfois. Pour les anciens termes transmis, essaimés jusque dans les langues plus lointaines, la marque grecque indique plutôt le transfert de vocables latins, formés dans le philhellénisme romain, qui devait absorber les exploits athéniens, et y succéder après synthèse - et le maintien ulté-

3. C'est le grand schème heideggérien. Cf., entre autres, Was ist das - die Philosophie?, Pfüllingen, Neske, 1956, p. 12. 
rieur d'un idiome grec perdant ses locuteurs et du coup spécialisé dans la diction technique du savoir. Sinon, le déroulement chronologique n'est nullement indice de l'origine. Difficile d'avancer que l'exercice de la philosophie n'est pas violemment, intensément, durablement influencé par le corpus grec. Encore cette empreinte est-elle fort diverse, selon que l'on se réfère à la mystique géométrico-arithmétique de Pythagore, à la geste de Diogène le Cynique, aux Problemata du Lycée, ou aux discours d'Isocrate - qui sans cesse plaça son éloquence sous le signe de philosophia et révoqua bruyamment la sophistique. Surtout, pourquoi en conclure un mot sur l'origine? Exactement, certains Anciens, dont Aristote, oui, assignaient ce début à ce qui n'était pas grec, Diogène Laërce écrivant pour résumer (et, lui, s'en offusquer) que «l'œuvre de la philosophie, certains disent qu'elle a commencé chez les Barbares $»^{4}$. Ce que ces auteurs grecs trouvaient de « grec » à la philosophie était la consolidation, la diffusion, la perfection; nul début. - La quête de l'origine, schème apparemment omnipotent, oh comme cette quête m'assomme, avec ses avatars multiples, de la mère patrie à papa-et-maman! Indéniablement, dans leurs activités, leurs écrits, leurs vestiges, des Grecs ont découvert et redéfini des pans entiers de ce que, comme d'autres, je considère dans le plus précieux de ma vie. La relation avec le commencement, avec l'acte de naissance est en revanche tout adventice. On pourrait soutenir qu'une discipline putativement nommée " philosophie occidentale » n'est qu'une suite de notes de bas de page aux textes de Platon ${ }^{5}$, sans que cela implique Platon comme authentique point zéro - car son œuvre est systématiquement traversée de multiples antériorités discursives et chronologiques, comme d'extériorités au principe grec (avec l'Égypte en particulier) - sans, a fortiori, prouver une genèse hellénique en général. Une hésitation, valait-il la peine de revenir sur cette non-origine aujourd'hui? Je le crains. Exemple parmi cent, même après avoir passé quelques décennies à réfléchir sur les Grecs et nous, Barbara Cassin que rien n'empêchera soudain de chanter en Homère «le [!] véritable [!!] commencement [!!!] de notre [!!!!] civilisation [!!!!!] $»^{6} ;$ bizarre...

4. Diogène Laërce, Vies des philosophes, I, 1. Sur ce que nous pouvons comprendre de la position d'Aristote, lire Fragmenta (Leipzig, Teubner, 1886), I, 1-26.

5. Pour paraphraser la célèbre formule d'Alfred North Whitehead, dans Process and Reality, Cambridge (Royaume-Uni), Cambridge University Press, 1929, III, I, 1.

6. Barbara Cassin, au début de sa préface à Richard Broxton Onians, Les Origines de la pensée européenne, Paris, Seuil, 1999. 


\section{Énième hellénique}

De la sainte naissance de nous à la tragique origine perdue (provoquant d'ordinaire la Chute de la modernité), les-Grecs se retrouvent ici enkystés dans une histoire qu'organise l'unitarisme du mythique Occident ${ }^{7}$ (ou de la drolatique Europe; j'y reviens): forcés de conférer un sens à une incroyable légende, depuis l'inclusive position d'exclusion que procure un simulacre de primauté absolue.

[4] Le passéisme s'en accommode à l'occasion, ou s'il le faut se défait de pareilles prétentions. Pour les amateurs d'antiquités, chez qui c'est joli puisque c'est vieux (et qui en effet des philhellènes, et j'en suis, n'avouerait a minima son envie, niaise aussi, de l'ancien puisque c'est bien?), pour lui, pour elle, peut valoir la peine d'en rajouter explicitement, de sacrifier aux grands débuts. Souvent pourtant, l'essentiel va au chérissable lointain d'une impressionnante chronologie. Fascination du temps reculé. Tant mieux si l'on s'offre la palme (l'Iliade est la plus ancienne épopée conservée « en entier»), tant pis sinon (d'accord, les épisodes de Gilgamesh devancent « Homère » d'un millénaire au bas mot, mais il n'empêche, vingt-cinq à vingt-huit siècles c'est beaucoup). Sous sa forme bourgeoise, le passéisme en France dominait l'enseignement du latin-grec, dont l'association annonçait assez le souci nécessaire de l'ancienneté parce que. Il s'associait volontiers, dans le volet conservateur dont j'eus plus d'un témoignage au cours de mon existence d'écolier, à une recherche de l'origine hébraïque et un exhaussement du latin mi-colonial mi-ecclésial laissant la-Grèce dans une limbe prestigieuse, nimbée par intermittence de primautés plus relatives ou plus discrètes. Pour sa part, l'antiquaillerie savante, peu philologique, moins socialement bien, se signale par une dilection quasi exotique, qui explique au passage la portée de la méthode anthropologique. Dans ce cas, ou les autres que porte une version plus institutionnelle des « études grecques ", le passéisme autorise la spécialisation (au détriment de la compétence), la spécialisation la fragmentation de la pensée (contre le discontinu), la fragmentation la parlure et le ronron (au lieu d'un dire). Il restera sur les marges du codex universitaire le poinçon d'une nostalgie pour ces grands commencements qu'il ne faudrait plus trop déclarer; dès lors se lira sous le filigrane le souhait revendiqué d'étudier l'autrefois grec sous prétexte d'une utilité de principe, de son rôle crucial pour la tradition, de la nécessité de la science avançant de toute façon, ou, une fois a quia,

7. Il faut encore lire plus loin l'article de Pierre Savy. 
du ça me plaît ${ }^{8}$ - réponse finalement plus honnête, quoique circulaire et justifiant le goût par le goût.

[5] La remise en contexte est un passage obligé de l'archéologie oscillant entre l'origine et le prestigieux passé. Sous sa forme light, elle se cantonne au saupoudrage de realia, dans le genre de la collection « La Vie quotidienne... ». On incarne le monde ancien; les corps, les odeurs, les bruits s'ajoutent au grand péristyle froid; pourquoi pas? De même que savoir comment la récitation d'Homère se déroulait au fil des siècles, ainsi que le suggère Gregory $\mathrm{Nagy}^{9}$, a une forte incidence sur la composition du texte écrit. L'implication suivante, et qui ne tarde guère à poindre parmi les partisans, est l'équation de la substance historique avec le contexte réel, qui devient aussitôt déterminant pour tout. Or non seulement l'on ne saurait dépasser le niveau théorique de la reconstitution, avec son inévitable surgissement fantasmatique mâtiné de quelque science, mais en outre le risque est de virer à l'hystérie matérialiste du référent, faisant de chaque artefact le commentaire déictique de son lieu social d'émergence. La forme non dégraissée du contextualisme oublie par exemple les effets de sens du texte théâtral car elle les a liquidés d'avance, au profit d'une laborieuse et fictionnelle description de la représentation. Nulle fatalité, je l'admettrais volontiers, il semble cependant si difficile de ne pas filer trop loin. On commence par se figurer, sur la foi de témoignages écrits, d'objets retrouvés dans les fouilles, de ruines, les « conditions de production », et qui poussent vers la performance comme rite et répétition. Puis on explique les œuvres en fonction de ce que l'on imagea.

[6] En ces parages, il est tout à coup patent que le plus fort degré de détermination historique n'efface pas l'actualisation. Celle-ci, même sous le signe d'un intensif déni, rend possible la prononciation de l'histoire. Isoler un contexte veut bien dire l'isoler du « nôtre », soit le faire contraster avec d'autres scènes, que l'on identifie et clôture, à commencer par cet hic et nunc « comparant l'incomparable $»^{10}$. Le célébrant de l'origine parle au présent de ce qui fut perdu ou perdure, voyez Agamben. Le

8. Dans un beau moment de comique sorbonnard tel que je m'en souviens, c'est à peu près ce que répondit Philippe Brunet pour expliquer son obsession de la métrique à Jean Bollack qui le pressait de se faire plus bavard, lors d'une journée d'études tenue à l'EHESS en septembre 2004.

9. Cf. par exemple ses Homeric Responses, Austin, University of Texas Press, 2003.

10. Titre désormais célèbre de l'ouvrage de Marcel Detienne, paru à Paris, au Seuil, en 2000. Lire les remarques de Klas Molde sur Detienne dans ce numéro de Labyrinthe. 


\section{Énième hellénique}

prêtre de la tradition vit un présent déporté vers un hier qui le soutient et le manipule, songez à Gadamer. Le chantre du glorieux passé maintenant présuppose le maintien éloquent de la nature humaine, rappelez-vous Romilly. On rencontre des actualisations d'apparence radicales, et sans reste, qui semblent s'affranchir de tout passé: Deborah Warner avec sa mise en scène de la Médée d'Euripide qui rendait brut le spectacle d'une violence et trahison érotiques, ou le type de discussion de plain-pied qu'une frange de la philosophie analytique peut entretenir avec Platon, avec Aristote, ou, pour mieux dire, leurs « arguments ». Il conviendrait de vérifier que cet actuel, nul dessein de reviviscence des débuts ne le visite, nulle restauration d'un passé plein ne le taraude (au bénéfice de ce que seraient le théâtre ou la philosophie) ${ }^{11}$. Quoi qu'il en soit, chaque circonstance de l'histoire pour les, ou des, Grecs anciens est mêlée, et à mi-mot souvent, à l'actualisation, fût-ce au nom de son contraire, ou d'une éternité (l'humaniste humanité des humanités) que l'on sent surtout depuis son chez-soi.

[7] Si ma parole est sardonique en l'occurrence, elle voudrait transcrire une méfiance à l'égard du déterminisme, de ses formes particulières, de l'illusoire issue que serait un pseudo-présent sans passé. Elle rend aussi le son d'une plus grande anxiété, annonçant l'auto-ironie, quand rien n'assure tout à fait que ces récupérations dogmatiques n'aient totalement tort, ni qu'il soit probable de s'en séparer convenablement. $\mathrm{Au}$ fond, les attitudes historisantes sont surtout irréfutables. Elles nous réduisent à l'état de l'interlocuteur subjugué dans les dialogues socratiques. - Athènes est l'origine de la démocratie moderne, parce qu'avant Athènes il n'est nulle démocratie (athénienne), et que les démocrates modernes parlent ensuite d'Athènes, et de démocratie. - Comment non? - Il faut regarder les Grecs comme appartenant au passé, ne pas les réduire à notre aujourd'hui, et nous interroger sur la différence de leur temps. - C'est indéniable. - Le propos d'un auteur ou ce texte de loi sont aussi les ramifications d'une civilisation, et les formes sociales signifient à leur tour. - Voilà qui est justement dit. - S'ils nous plaisent, nous ravissent, nous irritent, ces Anciens, ne trouvons-nous pas en eux les persistants éléments de notre existence? - Cela se pourrait, en effet.

11. De même que, symétriquement, le chantre du passé nonpareil opère forcément depuis son présent. Cf. les remarques des premier et dernier chapitres de Nicole Loraux, La Tragédie d'Athènes, Paris, Seuil, 2003, ainsi que les allusions au problème dans la préface de Jean-Pierre Vernant ouvrant le collectif $L a$ Grèce pour penser l'avenir, Paris, L'Harmattan, 2000, p. 16. 
Sauf s'il n'en est rien; mais alors que proposer? Au-delà des mauvaises raisons, à les dénombrer je me perdrais, se trouverait, qui sait, un bon motif pour garder quelque chose, sinon tout, de ces (dorénavant) quatre directions au cours de la navigation. Ces points cardinaux ne doivent pas situer le roc dogmatique, l'îlot du spécialiste auquel s'arrimer, ils orienteraient la course simplement, sans conditionner le trajet de ce point-ci à ce point-là. Suite de la métaphore, être un nouvel Ulysse, qu'aucune Pénélope n'attend, que nul Télémaque ne recherche, et qui ne rentrera pas au bercail, qui plutôt continuera de voguer d'île en île, se sentant mal accueilli ou princièrement reçu, jamais complètement chez lui ${ }^{12}$. Il faut alors beaucoup de ruse dans cette odyssée sans retour. Du coup, ne seraient plus objets d'admiration le positionnement précis, impeccable, la pureté de la direction, non, deviendraient notre souci la sophistication, l'allure, le risque, la justesse du périple ${ }^{13}$. Notre époque possède des navigateurs rusés, dont le voyage nous coupe le souffle, et nous encourage à prendre le vent ${ }^{14}$. Ici et ailleurs, si nous continuons la métaphore jusqu'à l'exténuement stylistique, compte le dessin de la mobilité navigatrice; et certaines routes se croisent, d'autres pas. Il serait opportun de préciser les principes de ma propre traversée - je diffère pourtant légèrement, devant tenter d'aborder un dernier récif - ultime témoignage de la figure de rhétorique, dont le fil se casse à cette ligne ${ }^{15}$.

[8] La détermination des Grecs par l'histoire n'est pas que générale ou épistémique : elle sert magnifiquement une histoire en particulier, sous son

12. Autrement dit, je viserais la Grèce de Personne, qui fait allusion au titre d'un ouvrage de Jean Bollack, et s'en distingue aussitôt par le choix volontaire de la majuscule (et la référence à l'épisode d'Ulysse et Polyphème). Rechercher dans « la Grèce de personne » (avec une minuscule) une inappropriable « Grèce, rendue à elle-même » (quatrième de couverture de Bollack, La Grèce de personne, Paris, Seuil, 1997), ce n'est pas trop mon style.

13. Je suis prêt à pleinement reconnaître l'air deleuzien de ce parcours cartographique, à condition de remarquer en même temps la pénible ratification de l'entreprise généralisante et anthropologique à laquelle se livra Deleuze (cf. les p. 9sqq. de l'ouvrage coécrit avec Félix Guattari, Qu'est-ce que la philosophie? Paris, Minuit, 1991, à rapprocher de la contribution donnée au volume Nos Grecs et leurs modernes).

14. Hommage en particulier aux deux grands « hellénistes » que nous publions dans ce dossier, Pietro Pucci et Pierre Judet de La Combe.

15. Sans que cela puisse étonner dans une textualité dominée par l'expédition militaire de l'Iliade et les multiples retours (nostoi) dont l'Odyssée fut le paradigme, l'image n'est pas neuve du tout. Mentionnons le Voyage du jeune Anacharsis en Grèce de Jean-Jacques Barthélemy, qui servit de somme aux Lumières et au-delà, ou, beaucoup plus récemment Mémoire d'Ulysse, Paris, Gallimard, 1996, qui est sans doute le meilleur ouvrage de François Hartog, et dont les qualités dépassent la nécessaire allégeance à l'historicisme et à l'anthropologie de convention. 


\section{Énième hellénique}

mode double, celle de l'Europe ou de l'Occident. Sur ce plan, l'entreprise de Martin Bernal avec Black Athena n'est pas d'urgence à refaire, mais la méditer toujours s'impose ${ }^{16}$. Athènes continue de servir à représenter une postulation impérialiste. On peut minimiser le philhellénisme guerrier et pro domo qu'élaborent un film, une bande dessinée, un jeu vidéo, ou affirmer que les 300 de Frank Miller ${ }^{17}$ ne sont par exemple pas que jeton de propagande. Certainement, l'album de Miller est une extraordinaire et hallucinante reconfiguration d'un point de vue spartiate, dont nous avons peu d'évidences textuelles hors des fastidieuses élégies de Tyrtée: les 300 (je ne parle pas du film) sont ainsi le meilleur livre apocryphe de la littérature lacédémonienne. Indéniablement, cette bande dessinée, depuis les États-Unis et au-delà, contribue à reposer les choses grecques dans une lignée occidentale. La bibliographie indiquée par Miller montre une solidarité avec le fonds de commerce néo-conservateur qui définit sans cesse l'Occident par la Grèce, et la Grèce par l'Occident ${ }^{18}$. Ces positions ne sont pas du tout la malheureuse conséquence d'une vulgarisation indue, il serait trop aisé de les juger indignes du « Bois sacré cher aux arts et aux Muses » qu'est la gentille Université, quand elles motivent en masse professeurs et étudiants ${ }^{19}$. En France, Sylvain Gouguenheim, dont il fut récemment trop parlé, feignait d'instruire le vulgaire; c'est ainsi que l'on défendit son Aristote au Mont-Saint-Michel, dont le point minable d'historiographie (y avait-il de bons Blancs, aussi, qui lisaient le grec avant le treizième siècle?) entendait déboucher sur la révélation des racines grecques de l'Europe chrétienne ${ }^{20}$. La conclusion de ce piteux ouvrage entamait d'une belle et pure voix ce grand chant de jeunesse

16. Martin Bernal, Black Athena. The Afroasiatic Roots of Classical Civilization, New Brunswick, Rutgers University Press, 1987-2006, 3 vol. Cf., plus loin dans ce numéro, l'extrait des mémoires inédits de Martin Bernal.

17. Frank Miller, 300, Milwaukie, Dark Horse Comics, 1999.

18. Figure ainsi dans cette liste un ouvrage tel que The Western Way of War, New York, Knopf, 1989, de Victor Davis Hanson. Hanson promeut un modèle grec belliqueux et volontiers rural (cf. son The Other Greeks. The Family Farm and the Agrarian Roots of Western Civilization, New York, Free Press, 1995), par défense de l'Occident désormais également attaqué par le multiculturalisme, le postmodernisme et le terrorisme islamiste. Cf. encore Carnage and Culture. Landmark Battles in the Rise of Western Power, New York, Doubleday, 2001.

19. Et qui, par leur pouvoir d'attraction, aux États-Unis du moins, participent clairement au maintien relatif du nombre de majors en études anciennes dans les institutions Ivy League et autres.

20. Tel est le sous-titre du livre de Sylvain Gouguenheim, Aristote au Mont-Saint-Michel, Paris, Seuil, 2008. La section bibliographique de l'article du wikipedia français correspondant à l'ouvrage (consultée en mai 2010) donne l'essentiel des références textuelles de soutien et d'accusation parues dans les médias et ailleurs. 
«Le soleil d'Apollon illumine l'Occident », aussitôt glosé de la note plus ravissante encore « Apollon est un dieu d'origine grecque (et non anatolienne) $»^{21}$, passagèrement j'avais eu peur. Folklore occidentaliste il y a, dans les médias, les grands établissements, les campus. Comment s'en surprendre si l'origine agit toujours? L'origine de quoi, à propos? De la pensée, de la modernité, de l'humanité, de la civilisation? Les passéistes même choisissent leur passé, alors pourquoi pas "le nôtre ", hein, plus que celui des voisins? L'actualisateur saisit mieux son affinité avec ces drôles de caractères. L'ami extrême du contexte a plus d'embarras, il se méfie infiniment de l'Occidentalisme - pas forcément de l'Occident, puisqu'il y peut voir une forme de contexte englobant. Alors risque de sortir un pourquoi sociologique, le grec à cause de son usage naguère central à l'école républicaine, ce qui n'est pas si insensé (biographème: après tout, au collège, au lycée, j'aurais eu du mal à apprendre l'araméen ou le sanscrit, le chinois, l'hébreu ou l'arabe, ces langues ne s'y enseignaient pas), tout en restant court. Face à une telle densification politique muant « la Grèce ancienne » un enjeu national, impérial, colonial, racial, et si l'on pâlit de l'appropriation, ose-t-on assumer une raison autre qu'incidente (le socius) et subjective (le plaisir)? Je verrais d'autres formules supplémentaires, comme la chance d'un corpus textuel suffisamment large et suffisamment borné pour permettre une enquête multiple et implexe; un accident, qu'on a peine à penser. Ou la valeur - qui s'y aventure? - de ce qui s'y dit. Oh ce motif effraie, qui menace de faire chair à canon, et qui textualise; oui mais c'est peut-être ce que la Grèce antique nous donne encore de mieux aujourd'hui, et s'il est question de dire que tout se vaut dans l'espoir illusoire d'abandonner les hiérarchies, ne comptez pas sur moi. Ou encore, et très tangiblement, et à cause (imaginons) de ces potentielles qualités contingentes ou inhérentes, les choses grecques ont, et sont (contre-) référentielles pour tant d'auteurs, tant de peuples, tant de sociétés, que sans même aucune continuité, elles nous ont un air de famille, et une étrangeté. Le fameux Occident, et encore plus la bonne Europe, ne sont que les détails encombrants de la paradigmatique grecque, voire les vagues lieux d'habitation de certains admirateurs acharnés. Incidemment, le grec ancien, avec les œuvres qu'il a permises, n'est - sinon à la rigueur pour les locuteurs contemporains d'un idiome à la fois transmis, métamorphosé, refait - ce grec n'est pas

21. Cf. n. 1 de la conclusion du livre de Gouguenheim, commençant à la p. 197 . 


\section{Énième hellénique}

plus aux unes qu'aux autres. Pour les Athéniens du cinquième siècle, le nom de l'Europe servit à désigner les imprécises frontières géographiques d'un ensemble opposé à l'Asie, et en particulier pour placer les Grecs (et Athènes d'abord) à la tête d'un groupe hétérogène aussi ample que l'ennemi perse ${ }^{22}$. Cette stratégie politique et poétique, Isocrate l'énonce parfaitement un peu plus tard dans son Panégyrique. L'Europe ne vaut que pour la Grèce, et pour Athènes. De là, persévérer dans sa songerie panhellénique afin d'étendre à l'Europe, ou l'Occident, c'est d'abord rester sourd à une tactique rhétorique, en son nom paradoxal, et mal placer ses espérances. Puis, à l'évidence, on souscrit une fois de plus à la généralisation. Ainsi s'oublie la lancinante accusation contre l'arbitraire définitoire, parcourant entre autres Euripide, et combien peut être peu grec un Grec, ou si peu barbare la Barbare ${ }^{23}$.

[9] Quelle dérision que cette Europe rivée à son continent, rêvant incontinent sa gloire universelle. Des peuples, des fous, des aventuriers, des nations, des marginaux, des textes d'Europe furent envoyés par bateaux, par cargos, par avions, par voitures à tous ceux qui en voulaient et surtout n'en voulaient point - mais pas pour l'Europe, ou si rarement. L'Europe actuelle est méta-européenne, un commentaire sur la possibilité de $l$ 'Europe, dont la glose se perd entre un faux début (la revendication territoriale de certains Grecs) et une absence de fin. L'Occident, à force qu'il se dresse et s'invente des Orients et des Autres, a plus de substance, et la performativité perfectionne sa machinerie. Il n'empêche, que les Occidentaux (lesquels ?) soient plus grecs (comment?) que les autres (lesquels ?), cette hypothèse, dont chaque partie est inquantifiable, insondable, indiscernable, n'entraînerait en rien que les exclus de l'Occident fussent extérieurs à la grécité. Partant, jusque dans cette démente logique du coefficient ${ }^{24}$, les-Grecs ne seraient pas plus porteurs de l'Occident que d'autres (que les Autres). Conclusion provisoire: le potentiel philhellénisme

22. La symétrie est très évidente dans Les Perses d'Eschyle ou chez le poète Chœrilus de Samos, qui écrivit une épopée sur ce sujet (les vestiges de ses Persiques figurent au début des Epicorum Gracorum fragmenta édités par Gottfried Kinkel, Leipzig, Teubner, 1877). De là, l'expédition contre Troie sera ressaisie comme le conflit de l'Europe contre l'Asie, ce qui n'était pas le cas chez Homère.

23. C'est l'une des leçons d'Hécube, en particulier lors du face-à-face entre la reine déchue et Ulysse (premier épisode).

24. « Mesurer le degré [... d d'hellénisation » procure un « solide critère d'identification des civilisations médiévales, européenne et islamique », nous assure Gouguenheim, Aristote au Mont Saint-Michel, p. 19. Cf. la critique apportée sur ce point par Alain de Libera dans Les Grecs, les Arabes et nous, Paris, Fayard, 2009, p. 19. 
de l'Occident, cet Occident défalqué de tous ceux qui ne correspondent pas à ladite définition ${ }^{25}$, cette dilection apparaît sous le jour de son ultérieure facticité, comme une reterritorialisation par supplément.

[10] La paradigmatique offre une tout autre chance. Arrêtons d'avoir mal à la racine, cessons de repérer les influences. Dans une phrase très débattue dès l'Antiquité, Isocrate, donc, propose d' '« appeler du nom de Grecs les gens qui partagent notre formation intellectuelle plutôt qu'une commune nature biologique $»^{26}$. La fonction locale (athénienne) de la formule, qui survient dans un discours de domination panhellénique, ne lance pas à tout coup un éloge planétaire de la tolérance multiethnique. Sa portée toutefois est telle que nous puissions supposer qu'en effet seraient grecs des moments et figures et personnes, hors d'un temps restreint, d'un espace donné, d'une civilisation précise, et sous condition d'un enseignement adapté, d'une paideia dont le vif aujourd'hui serait par exemple l'immersion textuelle. À ce titre, et après des siècles de recherches harassantes sur la grécité (philologique, historique, historiale, culturelle, existentielle, matérielle, économique, politique, artistique, etc.), le paradigme se ferait métamorphique. Plus exactement, adieu à l'hellénisation présupposant l'identifiable épure appelée hellénité. Est grec ce qui puise dans une formation grecque afin de n'être plus grec. En d'autres termes, la paideia, la norme, la transmission, le majoritaire sont à chaque cran pris pour grec, afin que par ce biais quelque chose d'autre ait lieu, qui ne soit point grec au sens d'avant, et qui devienne aussitôt, exactement grec. Une telle dynamique, via Athènes, permettait une réinvention du disensus, qui, loin d'être l'unique logique sociale, favorisait aussi que les-Grecs fussent des Grecs. Au-delà des remparts, elle m'autorise, si je le veux, à être grec à mon tour, pour autant que la formation ne soit justement pas confondue avec l'histoire, ni avec l'héritage, la continuité, la rémanence, ou autres schèmes plus ou moins équivalents. L'innutrition vaudrait davantage. Aussi bien, rien spécialement ne nous oblige à être grecs, et le fait que la revendication se fasse de plus en plus discrète dans les partis politiques hors l'extrême droite signale un abandon de fait, qui répond aux mutations des pouvoirs.

25. Y compris les Grecs contemporains, exclus d'Occident, comme le montre la carte du monde que produit Samuel Huntington dans The Clash of Civilizations and the Remaking of World Order, New York, Simon \& Shuster, 1996.

26. Isocrate, Panégyrique, 50. J'en rajoute un peu avec le «nom » et le « biologique », afin de rendre «onoma » et « genos », qui apparaissent juste auparavant dans la phrase. 


\section{Énième hellénique}

[11] En retour, je voudrais des études historiques repensant la chose grecque comme, par exemple, bactrienne et athénienne. Le regain d'intérêt pour l'Afghanistan « hellénisé » n'entraîne pas un nouveau langage. Un tout récent catalogue d'exposition continue de faire entendre à propos du site de Aï Khanoum qu'y figurent des temples «que l'on peut à peine dire grecs » mais où étaient honorées des divinités « intimement liées au panthéon grec », que l'on continua dans cette ville de « parler et d'écrire un grec de forme pure », que dans les arts mineurs on employait une « technique grecque » avec quelque " ouverture aux traditions asiatiques », etc. ${ }^{27}$ Sommes-nous vraiment sortis de la manière dont Droysen posait la question au dix-neuvième siècle, lorsqu'il inventait le récit d'une hellénisation progressive lors de $«$ la première unification du monde $»^{28}$ ? Si l'on considère plutôt que cette sculpture « orientale » est aussi grecque, qu'est-ce que cela induit pour mon Athènes classique? Par symétrie, le débat, avec Bernal, sur les racines afro-asiatiques de la Grèce classique a ouvert (ou rouvert) énormément. La violence, l'arrogance ou l'indigence des réponses ont en outre révélé l'efficace politique des recherches de Bernal $^{29}$. En même temps, l'identification de racines - et, outre le soustitre, un volet étymologique occupe tout le dernier volume de Black Athena - ferme la possibilité de désignations autres, et en revient, très classiquement, aux quêtes d'origines. La caractérisation du grec non grec, si elle s'appuie sur une citation, détournée, d'un orateur attique, n'est que le cas aigu d'un problème vaste, et qui porte sur notre capacité à parler de rencontres, d'interactions, de contacts, de synthèses dans les parties du social-historique. En ces zones, les renforts méthodiques depuis un bon siècle furent le diffusionnisme (recouvrant souvent une translatio studiorum, ou un processus de civilisation), la considération d'aires culturelles (menant à des monadismes anthropologiques ainsi qu'au clash of civilizations), les études postcoloniales (où la domination est perçue comme à la fois niant les autres et les conservant dans une

27. Contribution de Paul Bernard dans Fredrik Hiebert \& Pierre Cambon dir., Afghanistan, Washington, National Geographic, 2008, p. 93, 95, 99 et 101.

28. Johann Gustav Droysen, Geschichte des Hellenismus, Darmstadt, Primus Verlag, 1998, vol. I, p. 442. (Cette publication reprend le texte de la seconde édition révisée de 1885.)

29. Cf. la somme polémique dirigée par Mary Lefkowitz \& Guy MacLean Rogers, Black Athena Revisited, Chapel Hill, University of North Carolina Press, 1996. Bernal a répondu dans Black Athena Writes Back, Durham, Duke University Press, 2001. 
relégation inférieure ${ }^{30}$ ). L'hybridité et le multiculturalisme, que théorisent un Homi Bhabha ou une Martha Nussbaum à grands coups d'allusions grecques $^{31}$, ne me semblent propices qu'à décrire les lieux synchroniques d'une relation coloniale pacifiée (pax imperatoris), avec, donc, une forme d'angélisme politique, et une faible vertu heuristique. Un après-coup méthodique de l'optique (post)coloniale dans l'évocation des contacts ne devrait pas régler le cas de la violence dans l'irénisme du tiers ou du multiple, ni tenter d'y lire une disparition de la domination. Mais pourquoi ne pas trouver, en plus de la relation de pouvoir, l'intenable et réussie position de l'hétérogène ou du composite? Plutôt que de tracer l'importation de la statuaire grecque dans la représentation de Bouddha, repenser ce qui, dans la forme de Praxitèle, est susceptible de se modifier sans rompre, et par ricochet reconnaître la non-grécité d'un idéal type prétendument hellénique. Au lieu de créditer l'Égypte d'un crédit grec, comprendre que les Athéniens étaient si égyptiens qu'ils pouvaient ensuite s'emparer du Nil sans tellement helléniser. J'ai, cela se sait, un scepticisme insinuant vis-à-vis de l'histoire, disons, standard. Je n'ai pas d'illusions sur mon programme, et ne cherche pas à liquider les enquêtes plus conventionnelles. J'avance malgré tout qu'une relative nouveauté s'entendrait de reprendre le vieux débat sans influences, ni racines, ni respect mutuel, ni seulement domination-et-assimilation. Il reste là autant d'objets à créer, que de signification et de grammaire épistémique. Le contrecoup politique est manifeste, désarmer l'arraisonnement de la Grèce, enrayer l'enrôlement sous l'étendard occidental-européen, faire exploser la fallacieuse exposition à la civilisation: les Grecs n'étaient pas des Grecs, ou guère plus que ne l'étaient les habitants d'Égypte et de Bactriane. Plus, sous les conditions d'une formation à recommencer et renouveler sans cesse, la chose grecque est aussi à chercher dans ses revendications et « renaissances », dès qu'elles sortent de la tradition ou de la restauration: dans l'Haïti néo-classique, la Florence médicéenne, ou le monde d'Averroès.

30. Il vaut la peine de rappeler que, chez Droysen, l'hellénisation est bien vue comme une colonisation, et qu'elle est explicitement comparée aux efforts impériaux des grandes nations européennes du dix-neuvième siècle; cf. Geschichte des Hellenismus, vol. III, p. 428-434.

31. Respectivement Homi Bhabha, The Location of Culture, Londres, Routledge, 1994, et Martha Nussbaum, Cultivating Humanity, Cambridge (États-Unis), Harvard University Press, 1997. Sur le rapport entre le tiers genre platonicien et Bhabha, lire mon «Alter, inter », Labyrinthe, $\mathrm{n}^{\circ} 24,2006$. 


\section{Énième hellénique}

[12] Les défis de l'interprétation nous conduiraient à désassembler plus qu'une histoire, et atteindre le mythe de son ordonnancement. Place à la recomposition anachronique ${ }^{32}$. L'horizon se dégageant d'une nécessité occidentale, il est loisible de relier entre eux des points inattendus. De reconnaître une extériorité grecque, qui résiste à nos familiarités, et se distingue de nos usages dominants de réflexion, de sentiment, d'organisation. De saisir, immédiatement ou presque, combien nous sommes autant hors de nous, et que nous ne participons qu'incomplètement à ce grand corps doté d'une voix empruntée. De souligner que ni nous vraiment nous, ni les Grecs authentiquement grecs n'avons à nous croire. D'être alertés de l'impossible restitution de la chose grecque même, tout en recherchant des traces convaincantes de sa fiction. De lire bel et bien un passé révolu, de lire bel et bien au présent qui est à construire. De situer les agrégats de la tradition, par un désir de les mieux contredire. De ne pas céder aux désirs de tout remettre à plat, de lisser les aspérités, de tout prendre pour aujourd'hui. De vivre dans le texte et les détours de son langage. D'oser. Cette gymnastique de l'esprit - gymnastique, car exercice constant, et mise à nu de ce que je, nous, maintenant, hier, lui, elle, ceci, cela sommes - est la recomposition du disponible et la précipitation de « notre » temps comme de celui des, d'un, de plusieurs « Grecs » dans un chronique anachronisme, qui, par la précaution de l'entraînement, ne soit pas simple éclectisme. La philia n'est pas l'amitié, mais si la philia n'est pas non plus la philia, que se passe-t-il pour les amitiés que nous ressentons? Eh bien le récit de nos sentiments communique avec la description homérique d'Achille et Patrocle, ou Plutarque antidate Jankélévitch, et ce qui n'est pas pour nous nous revient ${ }^{33}$.

[13] Grâce à des Grecs parmi les Grecs, nous serions susceptibles de transgresser les conventions d'une histoire à reconfigurer, qui essaie de «nous » marginaliser comme Noires ou de « nous » inscrire indûment comme Blancs dans un récit identitaire. Nous pourrions dès lors nous emparer des bribes, des fragments de l'hier aujourd'hui comme hier et

32. Avec brio, Nicole Loraux plaida en la matière « pour une pratique contrôlée de l'anachronisme » (La Tragédie d'Athènes, p. 179). J'hésite à me placer sous cette tutelle, car la recomposition, si elle est bien limitée - ne procédant ainsi pas du sans-fin de l'incontrôlable-n'est cependant nul contrôle à tout coup, devant arrêter, surveiller, vérifier, évaluer, mesurer. À se demander si la «pratique contrôlée » n'a pas surtout pour but d'amortir le dérapage qu'est précisément l'anachronisme pour l'historien, bref d'annuler par avance le risque d'une sortie de piste et d'en offrir l'analogie à la place.

33. Ou comment situer en deux phrases mon livre À force d'amitié, Paris, Hermann, 2009. 
aujourd'hui. Une manière de dégonder le temps, ou le dégourdir. Ce qui nous resterait à dire, dans une autre rencontre hellénique. À ne considérer que quelques siècles d'un savoir écrit en grec, et dont certaines normes se sont répétées, ossifiées dans le cours moderne des habitudes disciplinaires, il saute aux yeux que nos récents savants ont trop oublié d'écrire. Hors les exceptions, que l'après-68 portait, mais progressivement tues ou adoucies, la voix protocolaire de la connaissance dans les sciences humaines favorise surtout trois poétiques: l'apodictique, la rhapsodie, la narration. La causerie, vivace encore, est plus la phase terminale de l'Intellectuel académique, notamment en France, en Italie. Sinon, l'apodictique prime dans l'Europe continentale, et la rhapsodie se tolère mieux dans l'université de langue anglaise; tendances temporaires, réversibles. La narration circule dans l'histoire et perfuse les autres genres. Au-delà du traité démonstratif en trois parties, du collage et rapiéçage d'articles monomaniaques, de la diégèse avec notes de bas de pages, la place est mince pour qui ne s'y colle pas, et les maisons d'édition peu aventureuses. Dans une parlure institutionnelle française, on entend même que la seule « forme recherche » c'est l'apodictique, que le reste n'est qu'essayisme reniant l'un des acquis scientifiques de la lutte des classes. Aux États-Unis, peu de scholars se mettent à une écriture autre ou surtout l'afficheraient, tant la rhétorique du déroulement textuel (la joke ou l'anecdote personnelle pour commencer, le I would argue that, le tissage de deux références théoriques, les fausses réponses de la fin), voire de l'agencement des morceaux, est professionnalisée $e^{34}$. Alors, mesurons-nous à l'impressionnante multiplicité poétique grecque en matière de preuve, de savoir, d'avancement de la pensée - cette multiplicité que seule une industrialisation de la connaissance via l'impérialisme universitaire a mise au pas, et encore fort récemment. Regardons ces textes grecs comme un remarquable répertoire discursif ${ }^{35}$. Allez, qui veut se lancer au-delà des trois ou quatre formes usuelles, dans : le dialogue, la lettre, le poème, le sille, l'éloge, la maxime, le fragment, l'apopthegme, le dit et action, l'écriture allitérative, le paragramme, la prosopopée, la

34. Cf., pour un salutaire contrefeu, le travail de Brian Lennon (par exemple « The essay, in theory », Diacritics, 2008, 38-3).

35. Il y aurait à dire sur le répertoire formel, en général (des formes de vie aux régimes politiques à la plastique). Il paraît parfois plus difficile, en ces parages, de ne pas succomber à un esthétisme purifiant, ou à une benoîte célébration (comme si c'étaient vraiment les Grecs, par exemple, qui inventèrent la démocratie ou la ville...). Mais bon. 


\section{Énième hellénique}

palinodie, la parodie, l'apologie, le « mythe », le caractère, l'aphorisme ou le discours? Et, davantage, cette inventivité de l'exposé doit nous inciter à tenter d'autres registres, disponibles dans leur non-usage jusque-là. Le but serait d'entretenir cette étrangeté qu'Aristote assigne au style dans la Rhétorique (III, 2), cette impression de léger déplacement dans les mots de notre dire et qui devrait justifier que l'on publie, au lieu de mâcher le déjà-connu. C'est aussi cela notre hellénisme, un décalage de l'écriture, qui devient xenè, notre invitée familière, ainsi qu'en moi des Grecs sont comme chez eux. Bref, il est encore temps de redonner voix aux choses grecques, de toutes les manières, de toutes les façons - sur tous les modes et tous les tons. Ou: par les Grecs, parlons.

[14] Alors, mes amies, mes compagnons, à ces conditions peutêtre deviendrons-nous encore aussi grecs que possible, c'est-à-dire pas vraiment, pas assurément, pas exactement; soit pas du tout en fait. Il m'arrive d'enrager devant cet énième éloge de ce qui n'exista pas ou cette parfaite reconstitution historique qui banalise au nom de la science. Et, je le disais d'abord, je me prends sinon à imaginer qu'après tant de temps à tenter de penser, en grec, et à des Grecs, je devrais avoir en récompense comme une saisie plus simple, au moins privilégiée - qu'il ne soit pas besoin de diffracter les lieux, de dilater les instants, de recomposer, de réinventer, depuis la base de mon vocabulaire à la qualité du style que permet ma réflexion. Je ne dois pas être le seul dans ce cas, à ressentir une nostalgie du plus léger, ce pourquoi je vous appelle en ce paragraphe. Pourtant l'époque que je crois la mienne, reconnaissez-la si vous voulez, n'en est pas aux tables rases. Je redoute aujourd'hui la félicité de la naïveté, elle prête trop au déferlement contemporain de l'ignorance, qui sous couvert de l'information proliférante, mondiale, multi-culti, et dite accessible, annule pour mieux ânonner la même chose. Certes, tout cela est compliqué, mais qui oblige qui à visiter la Grèce ancienne? La légitimité sociale s'est à peu près dissoute, avec elle hélas une certaine connaissance de la langue et des textes, tant mieux cependant pour l'opportunité de mieux lire que la déshérence devrait faciliter. Voilà en tout cas où je crois que nous sommes, quelque part entre le maintien d'un vestige de l'évidence et la nouvelle friche intellectuelle. Fort à parier que le désert soit en train d'avancer, permettant au moindre voyageur de paraître le meilleur guide, et rebutant la plupart des curieux. Pour celles, pour ceux qui voudraient insister, ce sera l'aride traversée, la mer ayant bien commencé de descendre. Il faut donc y croire plus que jamais, à 


\section{Labyrinthe, $n^{\circ} 36$}

cette occupation que presque plus rien ne justifie ni n'encourage, et qui ne signifie qu' au prix de nous faire parler et penser, contre la Grèce. Oui, des Grecs vraiment, tels que ni eux ni nous ne sommes ni n'avons été, ou: ce que nous finirions par n'être pas, à force d'être à eux, passionnément. 